\title{
DETERMINACIÓN DE MODELOS PARA EVALUAR EL CONSUMO DE POTENCIA EN TANQUES AGITADOS PARA SOLUCIONES PSEUDOPLÁSTICAS
}

\author{
Martín Berzosa Jiménez \\ Manuel Molina Córdoba
}

\begin{abstract}
Resumen
Esta investigación tuvo como objetivo determinar modelos matemáticos, que permitan evaluar la potencia requerida para agitar soluciones de naturaleza no newtoniana. Para este trabajo se utilizó un tanque estándar acrílico y un impulsor de hélice marina. A partir de glicerina como fluido newtoniano, preparada dentro del ámbito de viscosidad de 0,002 $\mathrm{Pa} \cdot \mathrm{s} \sim 1,07 \mathrm{~Pa} \cdot \mathrm{s}$, se establecieron los modelos de viscosidad tanto para la zona laminar como para la zona turbulenta para efecto de aplicarlos posteriormente cuando se trabaja con fluidos no newtonianos.

En una segunda etapa experimental se utilizaron como sustancias pseudoplásticas goma xantana, carboximetilcelulosa y goma guar, de las cuales se prepararon disoluciones a diferentes concentraciones correspondientes al ámbito de viscosidades de las soluciones de glicerina; se determinó para cada solución el consumo de potencia y junto con los modelos de viscosidad, se establecieron los modelos de consumo de potencia para flujo laminar y turbulento para cada una de las sustancias no newtonianas. La obtención de estos modelos permite la estimación de la potencia requerida en procesos de agitación a nivel de laboratorio y a partir de esta información puede procederse al cambio de escala para procesos industriales.
\end{abstract}

Palabras claves: modelos, consumo de potencia, fluidos pseudoplásticos, reología.

\begin{abstract}
The objective of this work was to determine mathematical models that will facilitate the evaluation of the potency required to stir non-newtonian solutions. A standard acrylic vessel and a marine helix impeller were used. Viscosity models for the laminar zone and turbulent zone were established from glycerine as a newtonian fluid within the viscosity range of $0,002 \mathrm{~Pa} \cdot \mathrm{s}-1,07 \mathrm{~Pa} \cdot \mathrm{s}$, to be applied later on non-newtonian fluids

Xantan gum, carboxymethylcellulose and Guar gum were used in a second experimental stage as pseudoplastic substances. These substances where prepared in different solutions at different concentrations corresponding to the viscosity range of the glycerine solutions. For each solution the potency consumption was registered and together with the viscosity models, consumptions models were established to laminar and turbulent flow for each of the non-newtonian substances. The obtaining of these models will allow us to estimate the potency required in stirring processes both at the lab levels initially, and scaled later for the industrial level.
\end{abstract}

Keywords: models, power consumption, pseudoplastic fluids, reology.

Recibido: 06 de noviembre del 2009 • Aprobado: 11 de febrero del 2010.

\section{INTRODUCCIÓN}

El proceso de agitación es una operación de importancia en la industria debido a que el éxito de muchas operaciones industriales depende de una agitación y mezcla eficaz (McCabe, 2001; Sharma, 2003).

En las bebidas que se preparan con extractos de frutas u otros, se agregan espesantes para mejorar la consistencia. Usualmente se utilizan gomas como carboximetilcelulosa, xantana y otras, las cuales provocan un comportamiento no newtoniano del fluido. En estos procesos es importante determinar la potencia que se requiere al agitar un fluido para efectos de diseño, dimensionamiento del equipo y para consideraciones económicas (Berzosa, 2008; Oldshue, 1983).

Los fluidos no newtonianos se caracterizan porque su viscosidad varía con el esfuerzo 
cortante aplicado (Chhabra \& Richardson, 1999; Puig, 2004). Esto origina dificultades para hacer una medición de la viscosidad de la solución en el momento en que se está agitando a determinada velocidad. Está dificultad fue resuelta en 1957 por Metzner y Otto quienes desarrollaron un método para tal fin. Estos autores establecieron que en primera instancia, se deben determinar modelos de viscosidad para flujo laminar y turbulento para un fluido newtoniano. Estos modelos son realmente correlaciones experimentales generadas siguiendo un procedimiento establecido, tal como se realiza en esta investigación. A partir de estos modelos, la viscosidad absoluta $(\mu)$ del fluido newtoniano se cambia a viscosidad aparente $\left(\mu_{\mathrm{a}}\right)$ para un fluido no newtoniano.

Esta investigación tiene como objetivo determinar modelos para el consumo de potencia en tanques agitados para soluciones pseudoplásticas. El propósito es que estos modelos estén en función de las propiedades físicas del fluido y de variables fácilmente medibles.

\section{FUNDAMENTOS TEÓRICOS}

\subsection{El método de Metzner y Otto}

En este método desarrollado por Metzner y Otto (1957) no se analiza la distribución de las fuerzas de cizallamiento dentro del tanque, sino que define una tasa media de cizallamiento para la suspensión a estudiar.

$$
\left(\frac{\mathrm{dv}}{\mathrm{dr}}\right)_{\text {prom }}=\mathrm{K} \cdot \mathrm{N}
$$

Donde:

$\mathrm{dv} / \mathrm{dr}$ : tasa media de cizallamiento

$\mathrm{K}$ : constante

$\mathrm{N}$ : velocidad de agitación

En este método el concepto de viscosidad aparente se basa en la utilización del sistema de agitación como un reómetro de proceso. Se define la viscosidad aparente media o efectiva como la viscosidad que tendría un fluido newtoniano si, al agitarlo bajo las mismas condiciones geométricas y operatorias, consume la misma potencia que el fluido no newtoniano (Puig, 2004).

El método consiste en la elaboración de una curva característica con un fluido newtoniano, representando el número de potencia en función del número de Reynolds en escala logarítmica. Una vez obtenida la curva característica se ajustan los datos a un modelo a partir del cual se puede despejar la viscosidad aparente dejándola en función de parámetros fácilmente medibles.

\subsection{Consumo de potencia en sistemas con agitación}

Para estimar la potencia, es preciso disponer de una correlación empírica de la potencia o del número de potencia en función de otras variables del sistema. Tal correlación se establece por análisis adimensional en función de los parámetros geométricos del tanque y del impulsor, además de parámetros como la viscosidad $(\mu)$, la densidad del líquido $(\rho)$ y las revoluciones por segundo. El número de potencia es función del número de Reynolds (Re) y del número de Froude (Fr).

$$
\mathrm{Np}=\mathrm{f}(\mathrm{Re}, \mathrm{Fr})
$$

donde:

$\mathrm{Np}=\frac{\mathrm{P}_{\mathrm{n}}}{\rho \cdot \mathrm{N}^{3} \cdot \mathrm{d}^{5}} \quad$ Número de potencia

$$
\operatorname{Re}=\frac{\rho \cdot N \cdot d^{2}}{\mu} \quad \text { Número de Reynolds }
$$

$$
\mathrm{Fr}=\frac{\mathrm{d} \cdot \mathrm{N}^{2}}{\mathrm{~g}} \quad \text { Número de Froude }
$$


Al graficar el número de potencia en función del número de Reynolds se obtiene la curva característica de donde se puede obtener la potencia consumida para agitar la solución según el régimen hidrodinámico en que se encuentre, tal como se muestra en la Figura 1.

Lo que dificulta la experimentación con fluidos no newtonianos es el hecho que la viscosidad varía con el esfuerzo de corte. Una manera para poder evaluar dicha viscosidad de una solución a determinadas condiciones de agitación es a través de un modelo matemático de viscosidad, obtenido para un fluido newtoniano, posteriormente dicha viscosidad absoluta se expresa como viscosidad aparente al utilizar la información para un fluido no newtoniano:

Se consideró el modelo mostrado en la ecuación (6).

$$
\mathrm{Np}=\mathrm{K} \cdot \operatorname{Re}^{x} \cdot \operatorname{Fr}^{y}
$$

Linealizando la expresión se obtiene la ecuación (7).

$$
\begin{array}{r}
\ln (\mathrm{Np})=\ln (\mathrm{K})+\mathrm{x} \ln (\mathrm{Re})+\mathrm{y} \ln (\mathrm{Fr}) \quad \mu=\mathrm{K} \cdot \mathrm{Pn}^{\alpha} \cdot \rho^{\beta} \cdot \mathrm{N}^{\gamma} \\
\mu=\mathrm{Pn}^{-1 / \mathrm{x}} \cdot \rho^{(1+\mathrm{x}) / \mathrm{x}} \cdot \mathrm{N}^{(3+\mathrm{x}+2 \mathrm{y} / \mathrm{x})} \cdot \mathrm{d}^{(5+2 \mathrm{x}+\mathrm{y}) / \mathrm{x}} \cdot \mathrm{K}^{1 / \mathrm{x}} \cdot \mathrm{g}^{-\mathrm{y} / \mathrm{x}}
\end{array}
$$

La que puede representarse como la ecuación (8).

$$
\mathrm{y}=\mathrm{a}+\mathrm{b} \cdot \mathrm{x}_{1}+\mathrm{c} \cdot \mathrm{x}_{2}
$$

Mediante regresión múltiple se obtienen los parámetros a, b, y c. Luego se puede desarrollar cada número adimensional obteniendo la ecuación (9).

$$
\frac{P n}{\rho \cdot N^{3} \cdot d^{5}}=k\left(\frac{\rho \cdot N \cdot d^{2}}{\mu}\right)^{x} \cdot\left(\frac{d \cdot N^{2}}{g}\right)^{y}
$$

Despejando la viscosidad se obtiene la ecuación (10).

Reagrupando se tiene la ecuación (11).

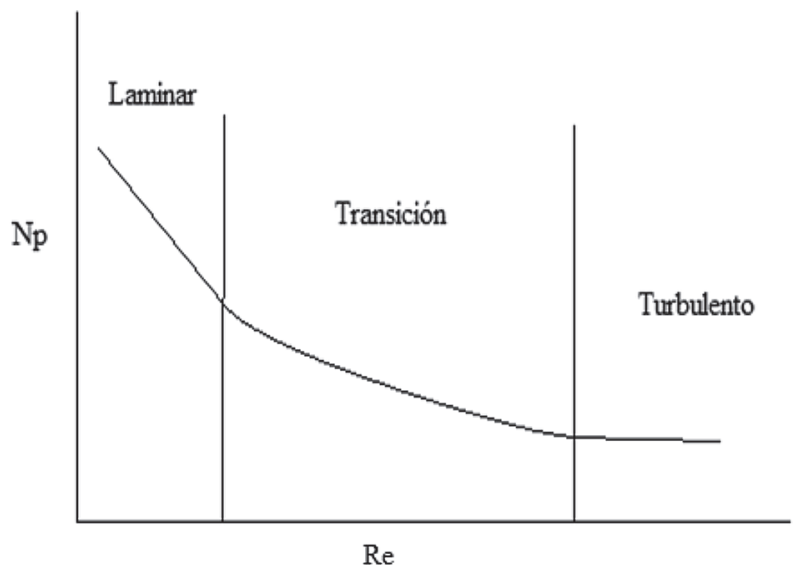

Figura 1. Regímenes existentes durante la agitación de un fluido.

Fuente: (Los autores). 
Donde:

$$
\alpha=-1 / x \quad \beta=(1+x) / x \quad \gamma=(3+x+2 y) / x \quad K=d^{(5+2 x+y) / x} \cdot k^{1 / x} \cdot g^{-y / x}
$$

Al aplicar el modelo de viscosidad, ecuación (11), a un fluido no newtoniano la variable viscosidad $\mu$ se sustituye por su equivalente de viscosidad aparente $\mu_{\mathrm{a}}$. La utilización del modelo de viscosidad se debe aplicar dentro del ámbito de viscosidades con las cuales se calcularon los parámetros del modelo utilizando un fluido newtoniano. Al contar con un modelo ajustado de acuerdo con la ecuación (11), se pueden calcular los números adimensionales para la solución no newtoniana y nuevamente con una regresión lineal múltiple, se obtienen los parámetros $\mathrm{k}, \mathrm{x}, \mathrm{y}$ del modelo mostrado en la ecuación (12).

$$
\frac{P n}{\rho \cdot N^{3} \cdot d^{5}}=k\left(\frac{\rho \cdot N \cdot d^{2}}{\mu_{a}}\right)^{X} \cdot\left(\frac{d \cdot N^{2}}{g}\right)^{y}
$$

Despejando Pn, se obtiene la ecuación (13).

$$
\mathrm{Pn}=\mathrm{k}^{\prime} \cdot \rho^{\mathrm{a}} \cdot \mathrm{N}^{\mathrm{b}} \cdot \mu_{\mathrm{a}}^{\mathrm{c}}
$$

\section{EQUIPO, MATERIALES Y METODOLOGÍA EXPERIMENTAL}

\subsection{Equipo experimental}

Tanque: el tanque para las pruebas está construido en acrílico y responde a las dimensiones de un tanque estándar. El diámetro interno $\mathrm{D}_{\mathrm{T}}$ es de 27 $\mathrm{cm}$, y satisface la relación $\mathrm{H} / \mathrm{D}_{\mathrm{T}}=1$. El volumen efectivo es de $16 \mathrm{~L}$. El tanque está provisto con cuatro deflectores, con un ancho de $2,2 \mathrm{~cm}$.

Impulsor: se utiliza un impulsor tipo hélice marina, con un diámetro equivalente de $8 \mathrm{~cm}$, colocado en el centro del tanque a una altura que cumple la relación $\mathrm{E} / \mathrm{D}_{\mathrm{T}}=1 /{ }_{3}$, donde $\mathrm{E}$ es la distancia del impulsor medida desde el fondo del tanque.
Equipo de agitación: para las corridas experimentales se utiliza el equipo Lightnin LabMaster $^{\mathrm{TM}}$ Mixer, este equipo permite registrar las revoluciones por segundo, el torque, la potencia y la temperatura del fluido mediante un sensor incorporado. Además en este trabajo se incorporó un sistema para la medición del amperaje (A) y el voltaje (V) para el cálculo de la potencia, con la finalidad de disminuir el ruido experimental.

Multímetro: en este trabajo se incorporó un sistema para la medición del amperaje (A) y voltaje (V) para el cálculo de la potencia, con la finalidad de disminuir el ruido experimental. Los resultados reportados en esta investigación corresponden a las mediciones realizadas por esta vía.

Viscosímetro: la viscosidad se mide con un viscosímetro de tipo Brookfield, con un ámbito de $1 \mathrm{cP}$ a $90000 \mathrm{cP}$.

\subsection{Materiales}

Se utiliza glicerina al $99 \%$ de pureza como fluido newtoniano. Como fluidos no newtonianos se utiliza goma xantana, goma guar, goma tragacanto y carboximetilcelulosa (CMC), todas de grado alimenticio.

\subsection{Metodología experimental}

a) Primera etapa: Determinación de un modelo para la viscosidad.

Se trabaja con glicerina como fluido newtoniano, para obtener un modelo (correlación experimental) para el cálculo de la viscosidad y, a partir de ella poder estimar la viscosidad aparente, siguiendo el procedimiento establecido por Metzner y Otto (1957); esta propiedad es intrínseca para el cálculo del consumo de potencia cuando se trabaja con fluidos no newtonianos. 
Determinación del consumo de potencia en el tanque vacío: se realizan mediciones registrando voltaje, amperaje, torque y potencia en el ámbito de 1,67 r/s a 21,67 r/s, logrando así determinar la potencia consumida para mover la masa del agitador y el eje.

Preparación de las soluciones de glicerina: se preparan soluciones a las siguientes concentraciones: 99, 95, 90, 85, 80, 60, 40 y $20 \%$. Con la glicerina al $99 \%$ se realiza la primera corrida; posteriormente se añade el volumen de agua necesario para diluir al $95 \%$; se agita durante un tiempo aproximado de $20 \mathrm{~min}$ a $600 \mathrm{r} / \mathrm{min}$ para homogeneizar la solución, seguidamente se realiza la corrida correspondiente. Se procede de la misma manera con las demás disoluciones en orden decreciente de concentración.

Determinación de la potencia consumida al agitar las soluciones de glicerina: una vez que se tiene la solución de glicerina homogenizada, se procede a agitar la solución en el intervalo de velocidades de $1,67 \mathrm{r} / \mathrm{s}$ a $21,67 \mathrm{r} / \mathrm{s}$. Para cada velocidad se registra el voltaje y el amperaje utilizando un multímetro, así como el torque y la potencia indicadas por el equipo Lightnin LabMaster ${ }^{\mathrm{TM}}$ Mixer. Entre cada cambio de velocidad se esperan 2 min antes de registrar los datos, con el fin de estabilizar el patrón de flujo. Una vez determinada la potencia bruta y la correspondiente en vacío, se determina la potencia neta con la ecuación (14).

$$
\mathrm{P}_{\text {Neta }}=\mathrm{P}_{\text {Bruta }}-\mathrm{P}_{\text {Vacío }}
$$

b) Segunda etapa: Determinación de un modelo para la potencia

En la segunda etapa se trabaja con tres fluidos no newtonianos para obtener un modelo, que permita calcular la potencia requerida para agitar soluciones de esta naturaleza.

Preparación de las disoluciones: los polímeros a utilizar son solubles en agua, pero requieren de tiempo para la hidratación de las moléculas, para ello, se disuelve la goma, se agrega lentamente para evitar la formación de grumos, se mantiene la agitación a una velocidad de $500 \mathrm{r} / \mathrm{min}$ y se deja reposar la solución para lograr la hidratación completa. Antes de realizar la corrida se agita la solución por un periodo de 30 min logrando así la homogenización. Se deja en reposo la disolución durante $15 \mathrm{~min}$ y luego se procede a realizar mediciones.

Cálculo de la viscosidad aparente: utilizando el modelo desarrollado con la glicerina, se calcula la viscosidad aparente para las diferentes velocidades de corte. Este modelo se aplica a todas las disoluciones no newtonianas preparadas en esta etapa.

Determinación de un modelo generalizado para la potencia: una vez obtenido un modelo para

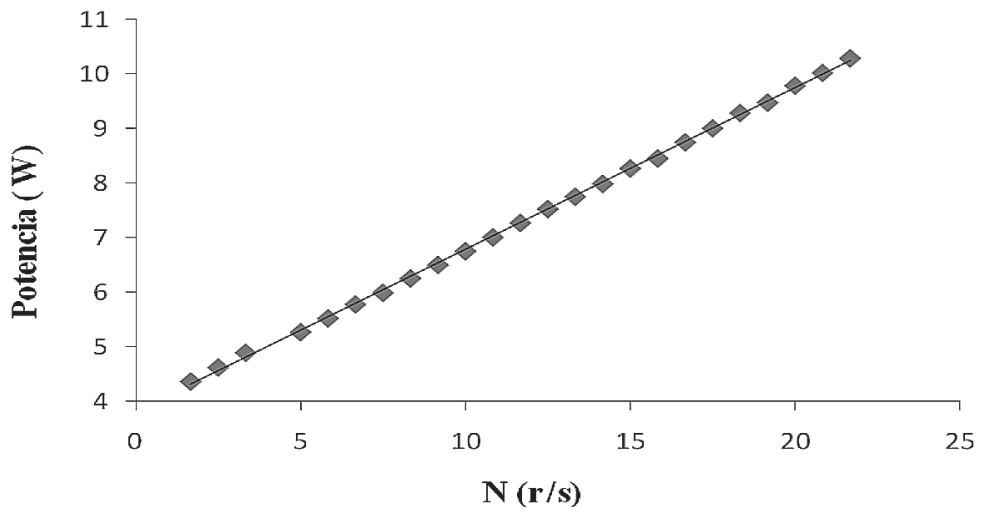

Figura 2. Potencia consumida con el tanque vacío.

Fuente: (Los autores). 
cada goma, se agrupan los datos de las tres gomas utilizadas y se realiza una regresión lineal múltiple para obtener los parámetros $k, x, y$ del modelo planteado en la ecuación (9). La potencia neta se obtiene de la ecuación (14).

Comprobación del modelo: se preparan disoluciones de goma tragacanto que estén en el mismo ámbito de viscosidades utilizado con las otras gomas, y se agitan registrando las variables de respuesta. Se compara la potencia obtenida experimentalmente con la obtenida a partir del modelo generalizado para la potencia y se analizan las desviaciones.

\section{ANÁLISIS DE RESULTADOS}

\subsection{Determinación de un modelo para la viscosidad}

Consumo de potencia requerido con el tanque vacío En la Figura2, se presenta el comportamiento del consumo de potencia respecto a la velocidad de agitación, sin utilizar un fluido en el tanque. Se encuentra un comportamiento lineal de la potencia respecto al aumento de las revoluciones.
Comportamiento de las propiedades físicas y consumo de potencia para las soluciones de glicerina

Para cada una de las disoluciones se determinó la densidad $\left(\mathrm{kg} / \mathrm{m}^{3}\right)$ y la viscosidad dinámica (Pa.s) a $25^{\circ} \mathrm{C}$, estos valores se muestran en el Cuadro 1.

En la Figura 3 se representa la evolución de la viscosidad en función de la concentración, se observa que existe un fuerte incremento de este parámetro para altas concentraciones de glicerina. A partir de la solución al $60 \%$, la viscosidad pasa de $1,42 \cdot 10^{-2} \mathrm{~Pa} \cdot \mathrm{s}$ a $1,07 \mathrm{~Pa} \cdot \mathrm{s}$ para una concentración del $99 \%$.

En la Figura 4 se muestra el comportamiento del consumo de potencia en función de la concentración de glicerina, se observa un incremento pronunciado para altas concentraciones de glicerina.

Se encuentra una similitud con la Figura 3, esto se debe a que al aumentar la viscosidad de las soluciones aumenta también el esfuerzo cortante sobre el agitador por lo cual aumenta el consumo de potencia.

Comportamiento del número de potencia respecto a la velocidad de agitación

En la Figura 5 se presenta el número de potencia en función de la velocidad de agitación

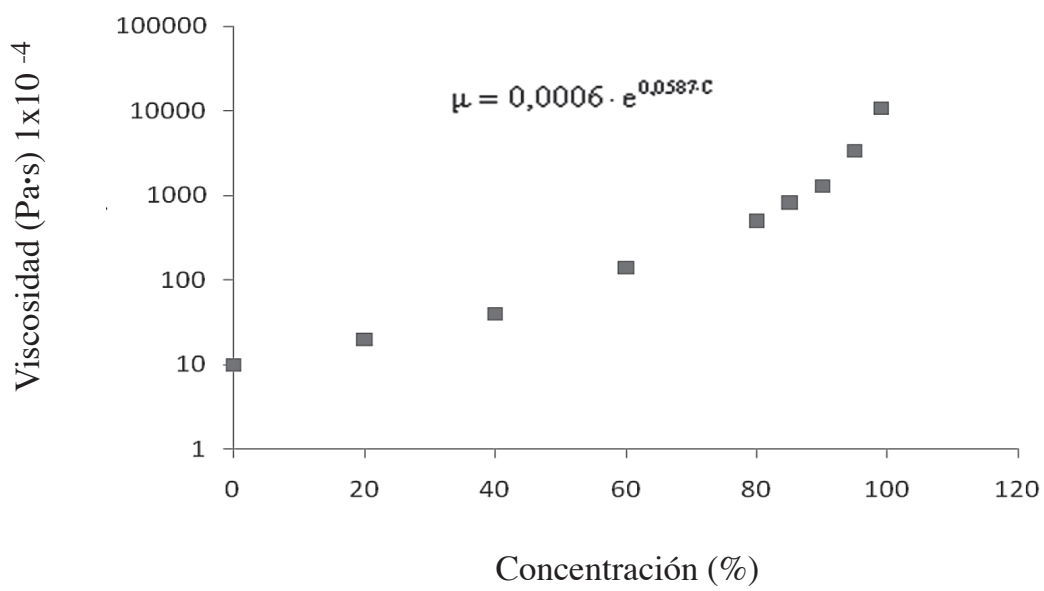

Figura 3. Evolución de la viscosidad en función de la concentración de glicerina.

Fuente: (Los autores). 
Cuadro 1. Densidad y viscosidad de las soluciones de la glicerina.

\begin{tabular}{lccccccccc}
\hline $\begin{array}{c}\text { Solución de } \\
\text { Glicerina } \\
(\%)\end{array}$ & $\mathbf{0}$ & $\mathbf{2 0}$ & $\mathbf{4 0}$ & $\mathbf{6 0}$ & $\mathbf{8 0}$ & $\mathbf{8 5}$ & $\mathbf{9 0}$ & $\mathbf{9 5}$ & $\mathbf{9 9}$ \\
\hline Viscosidad $(\mathrm{Pa} \cdot \mathrm{s}) \cdot 10^{4}$ & 10 & 20 & 40 & 140 & 500 & 830 & 1300 & 3400 & 10700 \\
Densidad $\left(\mathrm{Kg} / \mathrm{m}^{3}\right)$ & 998 & 1045 & 1092 & 1143 & 1191 & 1204 & 1216 & 1228 & 1238 \\
\hline
\end{tabular}

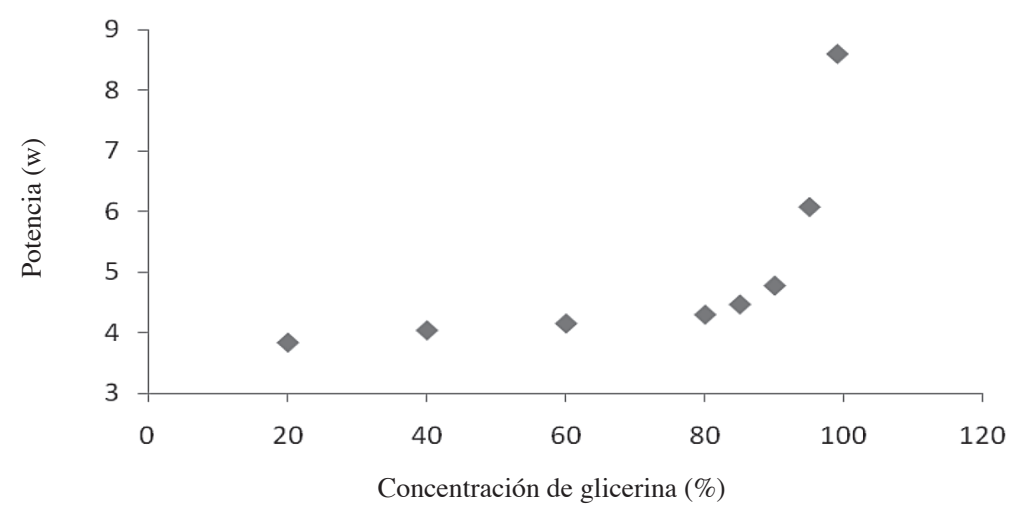

Figura 4. Evolución de la potencia neta en función de la concentración de glicerina.

Fuente: (Los autores).

para diferentes concentraciones de glicerina. Se pueden distinguir en las curvas dos zonas, la primera para $\mathrm{N}<10 \mathrm{r} / \mathrm{s}$, en donde las curvas poseen un decrecimiento pronunciado. Los valores más altos de $\mathrm{Np}$ se presentan para los esfuerzos cortantes más bajos. A estas velocidades de trabajo el fluido dentro del tanque se encuentra en régimen laminar y la variación en el consumo de potencia es sumamente sensible a los cambios en la velocidad de agitación, lo cual corresponde a lo reportado por Mc Cabe y Smith (2001) y Oldshue (1983).

La segunda zona, para $\mathrm{N}>10 \mathrm{r} / \mathrm{s}$, las curvas se mantienen práctimente constantes, lo cual indica según la teoría que el fluido dentro del tanque se encuentra en régimen turbulento y el consumo de potencia es independiente del aumento de la velocidad de agitación.

De las Figuras 4 y 5 se destaca una clara dependencia entre la viscosidad del fluido y la potencia consumida. Se observa que cuanto mayor es la viscosidad de la glicerina mayor es el número de potencia, obteniendo un valor máximo de 27,5 para una concentración del $99 \%, 23,3$ para una concentración del $95 \%$ y 17,2 para una concentración del $80 \%$.

\section{Ajuste del modelo para la viscosidad}

Utilizando números adimensionales se puede obtener un modelo que relaciona la viscosidad con las demás propiedades físicas del fluido. Al graficar el $\ln (\mathrm{Np})$ en función del $\ln (\mathrm{Re})$ se obtiene una curva linealizada donde se puede observar claramente que existen dos tendencias bien diferenciadas, tal como se muestra en la Figura 6.

Se encuentra que como las disoluciones menos concentradas poseen valores de Reynolds más altos, esto debido al factor viscosidad, ya que al disminuir éste, los números de Reynolds aumentan. 


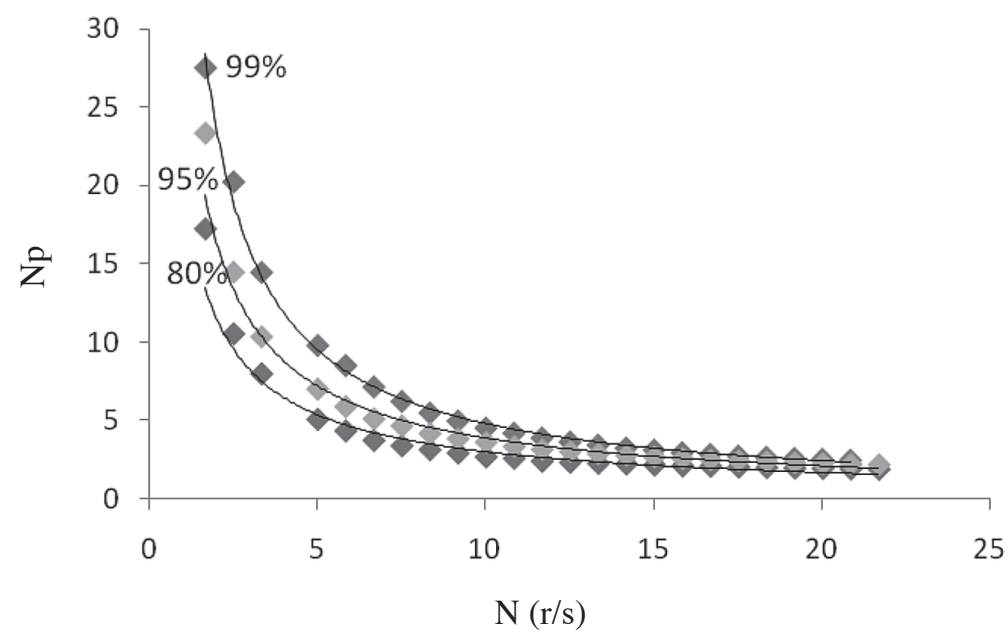

Figura 5. Evolución del número de potencia en función de la velocidad de agitación. Fuente: (Los autores).

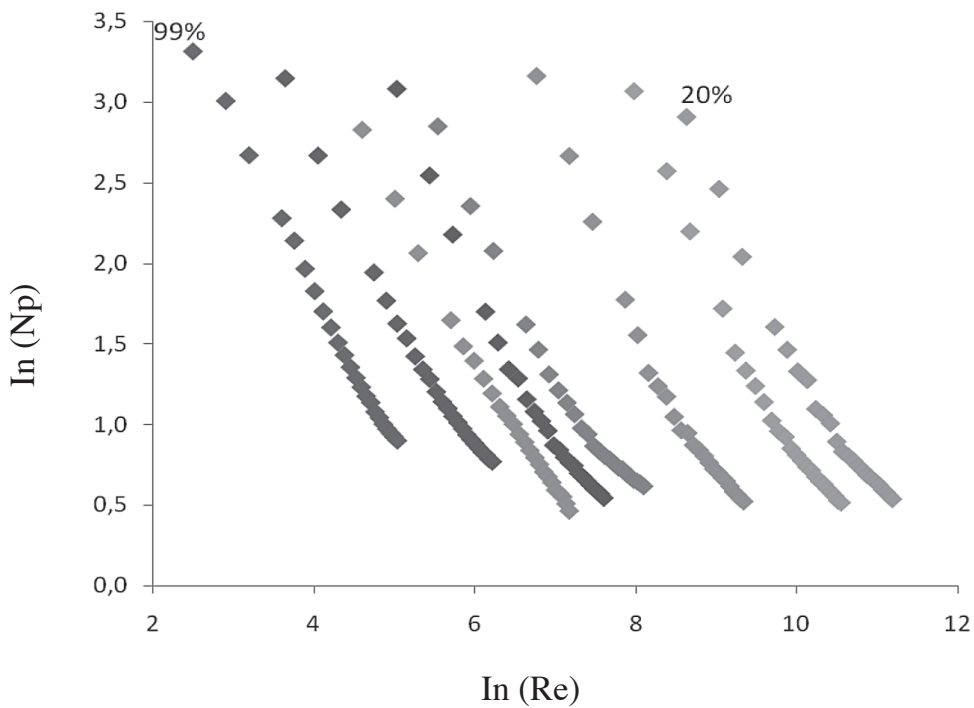

Figura 6. Curvas características linealizadas para las diferentes disoluciones. Fuente: (Los autores).

Esto es evidencia de la formación de dos zonas que corresponden a los regímenes originados al aumentar la velocidad de agitación.

Se considera que para velocidades entre $1,67 \mathrm{r} / \mathrm{s}$ y $8,33 \mathrm{r} / \mathrm{s}$ el régimen es laminar, entre
$9,17 \mathrm{r} / \mathrm{s}$ y $10,83 \mathrm{r} / \mathrm{s}$ se establece un régimen de transición donde no se ajustará a ningún modelo debido a su aleatoriedad de comportamiento. Y por último para velocidades en el intervalo de $11,67 \mathrm{r} / \mathrm{s}$ y $21,67 \mathrm{r} / \mathrm{s}$ el fluido se encuentra en régimen turbulento. 


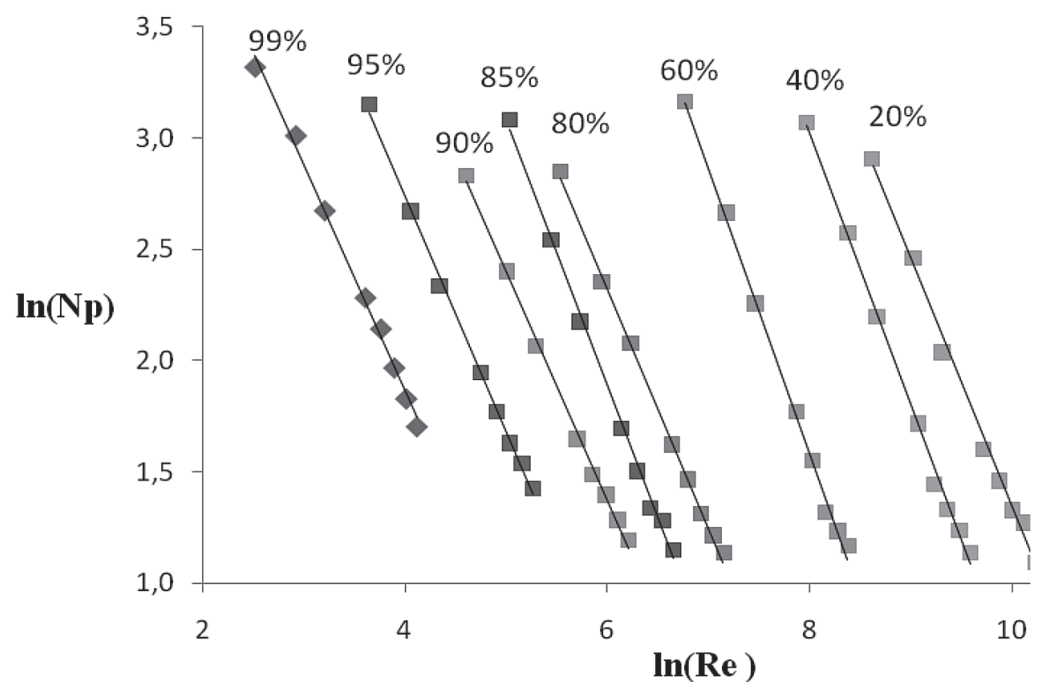

Figura 7. Curvas linealizadas para la zona laminar.

Fuente: (Los autores).

\section{Zona Laminar}

Al considerar los datos de la zona laminar se obtienen las curvas representadas en la Figura 7 , se encuentra que todas tienen una tendencia lineal y son bastante paralelas entre sí.

Con estas curvas se establece un modelo global de la forma $\mathrm{Np}=\mathrm{K} \cdot \mathrm{Re}^{\mathrm{x}} \cdot \mathrm{Fr}^{\mathrm{y}} \mathrm{y}$ mediante regresión lineal múltiple se obtienen los parámetros $K, x, y$ del modelo representado por la ecuación (6). Para el intervalo de disoluciones del $99 \%$ al $80 \%$ se obtuvo un ajuste con un coeficiente de correlación (r) de 0,99 y un error estandar $\mathrm{S}_{\mathrm{e}}$ de 0,08 el cual representa una variación en el segundo decimal respecto a los valores de $\ln (\mathrm{Np})$, si se amplía para considerar el ámbito de concentración el error estándar es de 0,3 , el cual se considera no aceptable. Al obtener un promedio de los valores, se determina que el error que se genera equivale a un $4 \%$, valor que se considera aceptable para el modelo obtenido y aplicable para velocidades entre $1,67 \mathrm{r} / \mathrm{s}$ y $8,33 \mathrm{r} / \mathrm{s}$. El modelo obtenido se muestra en la ecuación (15).

$$
\mathrm{Np}=9,25 \cdot \mathrm{Re}^{-0,203} \cdot \mathrm{Fr}^{-0,434}
$$

\section{Zona Turbulenta}

Al separar los puntos correspondientes a la zona turbulenta, es decir entre 11,67 r/s y 21,67 r/s; y al graficarlos se obtiene la Figura 8, en la cual se observa que los puntos poseen un buen ajuste con una línea recta aunque el paralelismo no es tan notable como en zona laminar. Las curvas para las concentraciones del $99 \%$ y del $95 \%$ son las que poseen los valores de $\ln (\mathrm{Np})$ más altos; al unir todos los primeros puntos de las rectas se forma una curva similar a la de la Figura 5. Este comportamiento responde a que las soluciones más concentradas poseen una mayor viscosidad $\mathrm{y}$ por ende se requiere mayor potencia para agitarlas.

Al hacer una regresión lineal que abarca todas las concentraciones se obtiene un modelo con un coeficiente de correlación de 0,88 y un error estándar $\mathrm{S}_{\mathrm{e}}$ de 0,25 los cuales no se consideran aceptables. La baja corelación obtenida en esta regresión puede deberse al no paralelismo entre las líneas. Para mejorar la respuesta se consideró el intervalo del $99 \%$ al $80 \%$ y se obtuvo el modelo representado por la ecuación (16).

$$
\mathrm{Np}=9,45 \cdot \mathrm{Re}^{-0,193} \cdot \mathrm{Fr}^{-0,415}
$$




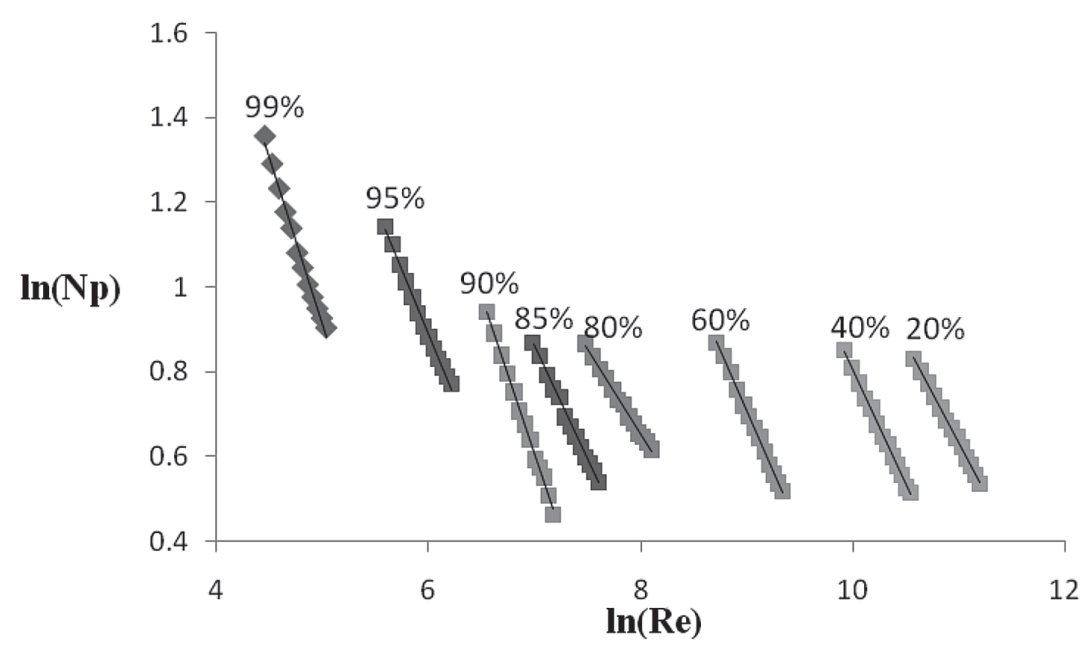

Figura 8. Curvas linealizadas para la zona turbulenta.

Fuente: (Los autores).

Se realizaron análisis de residuos correspondientes a las variables de Reynolds y Froude; a la vez se realizó un análisis de distribución normal donde se pudo observar la distribución de los residuos y su buen ajuste con la recta $\mathrm{y}=\mathrm{x}$ resultados no reportados en este trabajo; esto permite que con confianza estadística el modelo obtenido se pueda utilizar para cálculos posteriores.

De los modelos se despeja la viscosidad $\mu$ en función de las demás variables, como se muestra en las ecuaciones (17) y (18).

$$
\begin{aligned}
& \mu=3,44 \times 10^{15} \cdot \operatorname{Pn}^{4,93} \cdot \rho^{-3,93} \cdot N^{-9.50} \\
& \mu=4,77 \times 10^{16} \cdot \operatorname{Pn}^{5,18} \cdot \rho^{-4,18} \cdot N^{-10,24}
\end{aligned}
$$

Aplicando el método de Metzner y Otto se puede sustituir la viscosidad $(\mu)$, por la viscosidad aparente $\left(\mu_{\mathrm{a}}\right)$ y aplicar los modelos a fluidos no newtonianos.

Los modelos obtenidos en esta sección son congruentes con los reportados por Puig (2004), aun cuando las condiciones experimentales fueron diferentes.

\subsection{Determinación de un modelo para la potencia para fluidos no newtonianos}

En esta segunda etapa se tiene como objetivo obtener un modelo que pueda predecir la potencia neta requerida para agitar soluciones no newtonianas de carácter pseudoplástico.

Inicialmente, se pretendió cubrir el mismo intervalo de velocidades que se utilizó en los experimentos con glicerina, pero al utilizar velocidades superiores a las $16,67 \mathrm{r} / \mathrm{s}$, en las soluciónes se genera un régimen de alta vorticidad, provocando la incorporación de aire al sistema. Este fenómeno se encuentra principalmente en las soluciones de altas concentraciones (a su vez altas viscosidades) causando que el aire que entra produzca una mezcla bifásica, que provoca una disminución en el esfuerzo de corte y por ende, en la potencia requerida. Para las soluciones de goma guar y xantana el fenómeno aparece a velocidades de $15 \mathrm{r} / \mathrm{s}$, pero esto se debe a la diferencia de viscosidad entre las soluciones.

Comportamiento de la viscosidad aparente respecto a la concentración

La Figura 9 presenta la variación de la viscosidad aparente para diferentes 
concentraciones utilizadas y para una velocidad de 7,50 r/s correspondientes al régimen laminar. Se observa que la viscosidad aparente crece de manera potencial conforme aumenta la concentración.

Este comportamiento coincide con el de las soluciones de glicerina, cuando se compara la Figura 9 con la Figura 3, se observa que poseen tendencias similares; esto concuerda con el principio de Metzner y Otto en el cual se pueden relacionar el consumo de potencia para dos sustancias de naturaleza diferente si éstas poseen ámbitos similares de viscosidad.

En la Figura 10 se observa el comportamiento de la viscosidad aparente en función de la concentración para la zona turbulenta, para una velocidad de $13,33 \mathrm{r} / \mathrm{s}$. Se encuentra una tendencia exponencial; para la goma xantana es menos pronunciado, esto se debe al efecto del aumento en el esfuerzo cortante aplicado y además esta goma presenta un mayor carácter pseudoplástico.

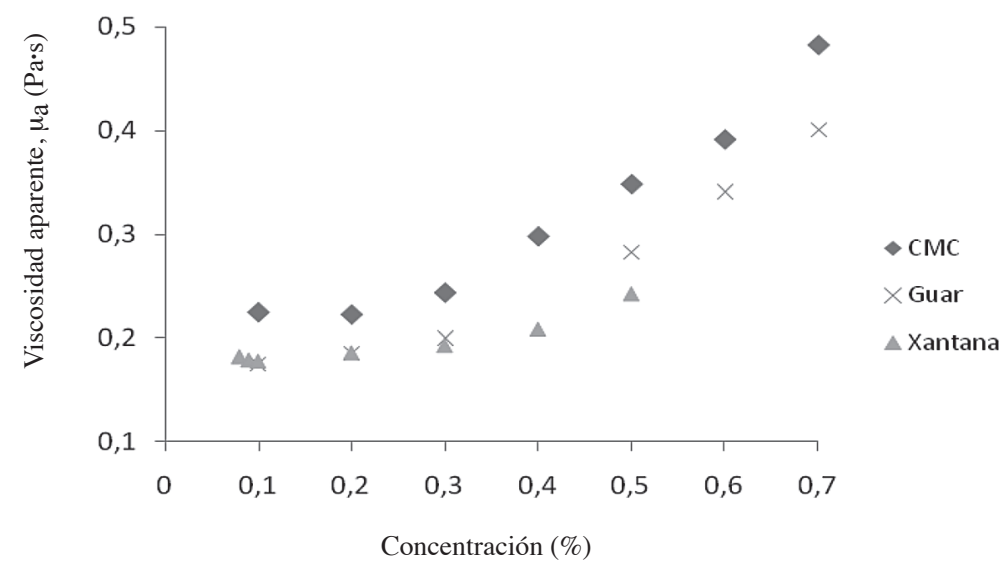

Figura 9. Variación de la viscosidad aparente con la concentración para las diferentes sustancias no newtonianas en la zona laminar.

Fuente: (Los autores).

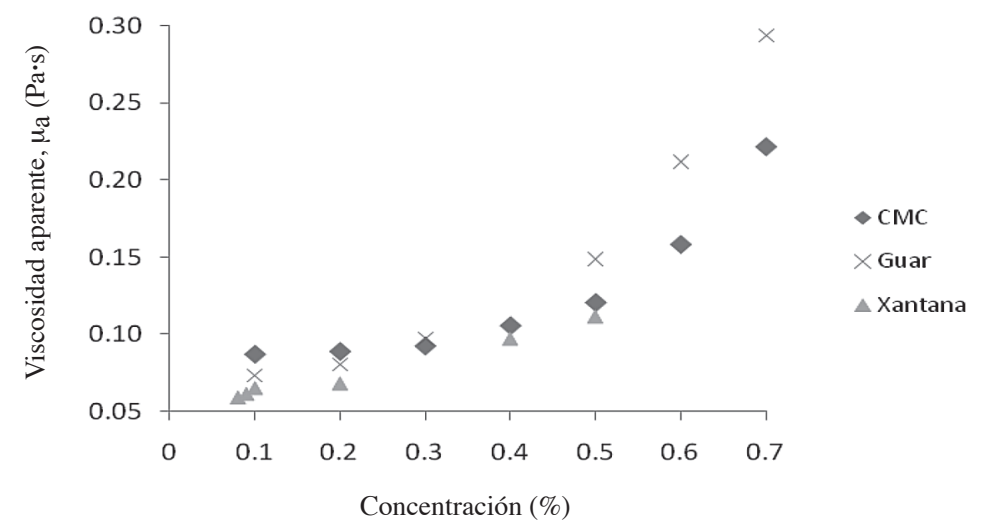

Figura 10. Variación de la viscosidad aparente con la concentracion para las diferentes sustancias no newtonianas en la zona turbulenta.

Fuente: (Los autores). 
Comparando las Figuras 9 y 10 se puede notar que la goma guar y la goma CMC poseen valores de viscosidad similares, de hecho a bajas concentraciones la curvas se cruzan, y los puntos se encuentran unidos entre sí en la zona turbulenta lo que indica que estas gomas también sufrieron cambios debido al aumento en el esfuerzo cortante.
Comportamiento de la potencia neta respecto a la viscosidad aparente

En la Figura 11 se presenta el consumo de potencia neto respecto a la viscosidad aparente, este comportamiento es originado por el aumento en el esfuerzo cortante necesario para agitar la solución.

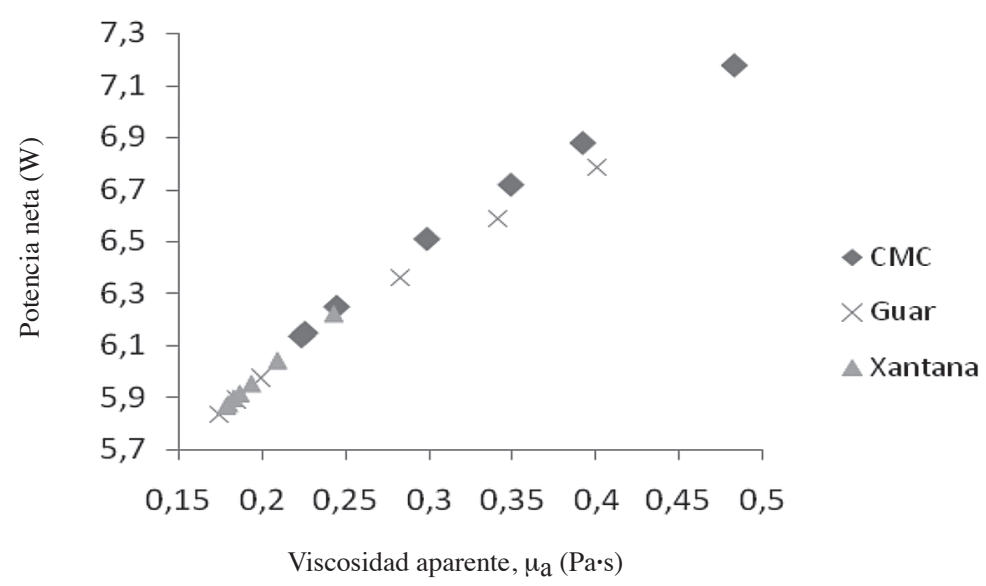

Figura 11. Evolución de la potencia neta con la viscosidad aparente para zona laminar.

Fuente: (Los autores).

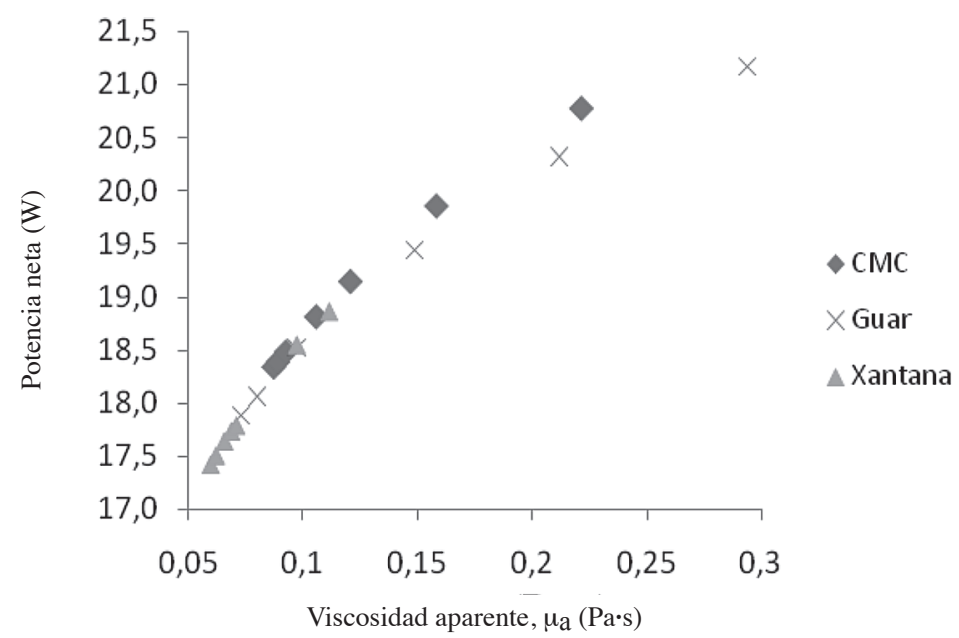

Figura 12. Evolución de la potencia neta con la viscosidad aparente para la zona turbulenta.

Fuente: (Los autores). 
En la Figura 12 se presenta el comportamiento de las mismas variables para la zona turbulenta; se puede observar que la tendencia es la misma que para la zona laminar.

Para ambas zonas las curvas podrían ajustarse de manera lineal, tal como lo presenta Puig (2004), pero basados en los resultados experimentales se logra un mejor ajuste al utilizar modelos potenciales. Al ajustar las curvas se obtienen las expresiones mostradas en el Cuadro 2.

\section{Ajuste del modelo para la potencia}

Se calcularon los números adimensionales de Reynolds (Re), Froude (Fr), y número de potencia (Np), y se ajustaron modelos de la forma $\mathrm{Np}=\mathrm{K} \cdot \mathrm{Re}^{\mathrm{x}} \cdot \mathrm{Fr}^{\mathrm{y}}$ para las zonas laminar y turbulenta para cada una de las gomas utilizadas. Los modelos obtenidos para la zona laminar y la turbulenta coincidieron para las tres gomas, obteniendo en cada una los mismos parámetros a través de una regresión.

Cuando se trabaja con gomas del mismo carácter reológico y en el mismo intervalo de viscosidades y velocidades, se espera que los parámetros de los modelos sean similares. Los modelos obtenidos para la zona laminar y turbulenta se presentan en las ecuaciones (19) y (20) respectivamente:

$$
\mathrm{Np}=9,536 \cdot \operatorname{Re}^{-0,202} \cdot \mathrm{Fr}^{-0,435}
$$

$$
\mathrm{Np}=9,426 \cdot \mathrm{Re}^{-0,193} \cdot \mathrm{Fr}^{-0,415}
$$

Para corroborar los modelos obtenidos se realizó un análisis de residuos (no reportado) para cada una de las gomas. Se encontró un comportamiento aleatorio lo que indica que no hubo sesgos durante la experimentación (Berzosa, 2008).

\section{Ajuste del modelo generalizado}

Con el objetivo de disponer de un modelo general de consumo de potencia de las sustancias pseudoplásticas utilizadas, se realizó una regresión múltiple global. Los modelos generalizados obtenidos son los mismos mostrados en las ecuaciones (19) y (20).

Los modelos para potencia se presentan en las ecuaciones (21) y (22) los cuales cumplen con el objetivo perseguido, ofreciendo una expresión para la potencia en función de variables fácilmente medibles.

$$
\begin{aligned}
& \operatorname{Pn}=7,073 \times 10^{-4} \cdot \rho^{0,797} \cdot N^{1,926} \cdot \mu_{a}^{0,203} \\
& \operatorname{Pn}=6,013 \times 10^{-4} \cdot \rho^{0,807} \cdot N^{1,977} \cdot \mu_{a}^{0,193}
\end{aligned}
$$

\section{Comprobación de los modelos}

Para corroborar los modelos, se utilizó goma tragacanto, sustancia que posee propiedades pseudoplásticas similares a las gomas utilizadas en esta investigación. Se realizaron dos corridas a concentraciones del $0,5 \%$ y del $0,6 \%$ registrando las mismas variables voltaje y amperaje en el

Cuadro 2. Modelos para potencia neta en función de la viscosidad aparente.

$\begin{array}{lcc}\text { Sustancia } & \text { Laminar } & \text { Turbulenta } \\ \text { CMC } & \mathrm{P}_{\mathrm{n}}=8,31 \cdot \mu_{\mathrm{a}}^{0,201} & \mathrm{P}_{\mathrm{n}}=25,4 \cdot \mu_{\mathrm{a}}^{0,134} \\ \text { Guar } & \mathrm{P}_{\mathrm{n}}=8,00 \cdot \mu_{\mathrm{a}}^{0,181} & \mathrm{P}_{\mathrm{n}}=24,5 \cdot \mu_{\mathrm{a}}^{0,120} \\ & \mathrm{P}_{\mathrm{n}}=8,10 \cdot \mu_{\mathrm{a}}^{0,187} & \mathrm{P}_{\mathrm{n}}=24,9 \cdot \mu_{\mathrm{a}}^{0,127}\end{array}$


ámbito de velocidad utilizado para los modelos generados previamente.

La viscosidad de las soluciones preparadas a esas concentraciones, fue menor comparada con las de goma xantana, CMC y goma guar. Al calcular la potencia neta consumida utilizando las ecuaciones (21) y (22) y compararla con la medida experimental los resultados muestran que la desviación para ambos modelos es inferior al $1 \%$ lo cual indica que los modelos son confiables si se utilizan dentro de las restricciones de velocidad, viscosidad y naturaleza del fluido.

\section{CONCLUSIONES}

1. Cuando se realiza el ajuste de los datos al modelo propuesto, es necesario separar los resultados en dos intervalos. Uno para velocidades entre $1,67 \mathrm{r} / \mathrm{s}$ y $8,33 \mathrm{r} / \mathrm{s}$ y otro para velocidades entre $11,67 \mathrm{r} / \mathrm{s}$ y $16,67 \mathrm{r} / \mathrm{s}$, que corresponden a los régimenes laminar y turbulento.

2. La viscosidad crece en forma exponencial con la concentración tanto para fluidos newtonianos como no newtonianos.

3. El efecto de la pseudoplásticidad o adelgazamiento debido al esfuerzo de corte, es más notable a altas concentraciones para todos los fluidos utilizados.

4. A partir de $16,67 \mathrm{r} / \mathrm{s}$ se introduce aire a las soluciones debido a la alta turbulencia y vorticidad, produciendo un sistema bifásico que afecta el sistema disminuyendo la potencia consumida.

5. Utilizando glicerina como fluido newtoniano se obtuvieron los siguientes modelos para la viscosidad, los cuales pueden expresarse posteriormente como viscosidad aparente para aplicarlos a sistemas no newtonianos dentro de los ámbitos de velocidades indicados.

$\mu=3,44 \times 10^{15} \cdot \operatorname{Pn}^{4,93} \cdot \rho^{-3,93} \cdot \mathrm{N}^{-9.50}$

$[\mathrm{N} ; 1,67 \mathrm{r} / \mathrm{s} \sim 8,33 \mathrm{r} / \mathrm{s}]$ $\mu=4,77 \times 10^{16} \cdot \operatorname{Pn}^{5,18} \cdot \rho^{-4,18} \cdot \mathrm{N}^{-10,24}$

$[\mathrm{N} ; 11,67 \mathrm{r} / \mathrm{s} \sim 21,67 \mathrm{r} / \mathrm{s}]$

6. Utilizando goma xantana, CMC y goma guar en la preparación de fluidos no newtonianos se obtuvo en este trabajo los siguientes modelos generalizados para el cálculo de la potencia neta consumida, aplicables dentro de los ámbitos de velocidades de trabajo.

Pn $=7,073 \times 10^{-4} \cdot \rho^{0,797} \cdot \mathrm{N}^{1,926} \cdot \mu_{\mathrm{a}}^{0,203}$

$[\mathrm{N} ; 1,67 \mathrm{r} / \mathrm{s} \sim 8,33 \mathrm{r} / \mathrm{s}]$

$\operatorname{Pn}=6,013 \times 10^{-4} \cdot \rho^{0,807} \cdot \mathrm{N}^{1,977} \cdot \mu_{\mathrm{a}}{ }^{0,193}$

[N; 11, $67 \mathrm{r} / \mathrm{s} \sim 16,67 \mathrm{r} / \mathrm{s}]$

7. El análisis estadístico realizado a cada uno de los modelos no revela ninguna tendencia específica, excluyendo la posibilidad de errores sistemáticos en las etapas experimentales de la investigación.

8. Para aumentar la confiabilidad y disminuir el ruido experimental en esta investigación, se introdujo la utilización de un multímetro para la medición de las variables voltaje y amperaje.

9. Los cambios en la metodología, respecto a otros autores permite reducir el ruido experimental y, a su vez se dispone de este recurso para su aplicación a otros fluidos de naturaleza diferente.

10. La comprobación de los modelos utilizando goma de tragacanto muestra la confiabilidad de los modelos obtenidos.

11. Los modelos de consumo de potencia aportados en esta investigación para fluidos pseudoplásticos permite la estimación de la potencia requerida en procesos de agitación a nivel de laboratorio y a partir de ella puede procederse al cambio de escala para procesos industriales aplicando el criterio de intensidad de agitación (potencia/volumen). 


\section{REFERENCIAS BIBLIOGRÁFICAS}

Berzosa, M. (2008). Evaluación del consumo de potencia en tanques agitados para sistemas no newtonianos en fase acuosa. Proyecto de graduación para optar por el grado de Licenciatura en Ingeniería Química, Universidad de Costa Rica, San José, Costa Rica.

Cavadas, A. \& Pinho, F. (2001, noviembre). Power consumption and circulation flow rate of polymer solutions in a stirred vessel powered by a hyperboloid impeller. En: ASME International Mechanical Engineering Congress \& Exposition. New York, USA.

Chhabra R. \& Richarddson, J. (1999). Nonnewtonian flow in the process industries. Great Britain: Butterworth-Heinemann.

Mc Cabe, W. \& Smith, J. (2001). Operaciones unitarias en Ingeniería Química. México: Mc Graw-Hill.Metzner, A. \& Otto R. (1957). Agitation of non-newtonian fluids. AICHE Journal, 3(1), p. 3-10.
Oldshue, J. (1983). Fluid mixing technology. New York: Mc Graw-Hill.

Puig, J. S. (2004). Avaluació del procés de desintegració de papers recuperats. Proyecto de graduación para optar por el grado de Doctor en Ingeniería Química, Universidad de Girona, Girona, España.

Sharma, S. (2003). Ingeniería de alimentos. México: Limusa Wiley.

\section{SOBRE LOS AUTORES}

\section{Martín Berzosa Jiménez}

Ingeniero Químico

Teléfono: (506) 2438-0605

Facsímil: (506) 2582-1482

Correo electrónico: iberico2002@gmail.com

\section{Manuel Enrique Molina Córdoba}

Escuela de Ingeniería Química, Universidad de Costa Rica.

Teléfono: (506) 2511-3421

Facsímil: (506) 2225-5622

Correo electrónico: emolina@fing.ucr.ac.cr 
